\title{
Non-reflecting boundary condition on ellipsoidal boundary
}

\author{
Hélène Barucq ${ }^{* \dagger} \quad$ Anne-Gaëlle Dupouy St-Guirons ${ }^{\ddagger \dagger *} \quad$ Sébastien Tordeux ${ }^{* \dagger}$
}

October 2, 2011

\begin{abstract}
The modeling of wave propagation problems using finite element methods usually requires the introduction of a fictitious truncating surface enclosing the computational domain around the scatterer of interest. Absorbing boundary condition are classically considered to avoid spurious reflections. In this paper, we investigate some properties of the Dirichlet to Neumann map posed on a spheroidal boundary in the context of the Helmholtz equation to efficiently deal with an elongated scatterer.
\end{abstract}

\section{Introduction}

In the context of time-harmonic linear acoustic wave propagation, a classical problem consists in considering the scattering by an impenetrable bounded rigid body $\Omega^{\prime} \subset \mathbb{R}^{3}$ with regular boundary illuminated by an incident pressure wave $u_{\text {inc }}(\mathbf{x})=\exp (i \mathbf{k} \cdot \mathbf{x})$. The wavenumber $k=\sqrt{k_{1}^{2}+k_{2}^{2}+k_{3}^{2}}$ is the norm of the wave vector $\mathbf{k}=\left(k_{1}, k_{2}, k_{3}\right)$. The phasor of the pressure field $u_{\text {tot }}$ is then governed by a well posed problem [13] composed of a Helmholtz equation equipped with Neumann boundary condition. The scattered field $u(\mathbf{x})=u_{\text {tot }}(\mathbf{x})-u_{\text {inc }}(\mathbf{x})$, with $\mathbf{x} \in \Omega=\mathbb{R}^{3} \backslash \overline{\Omega^{\prime}}$ solves the following exterior problem:

$$
\left\{\begin{array}{l}
u \in H_{\mathrm{loc}}^{1}(\Omega)=\left\{u: \Omega \longrightarrow \mathbb{C} \mid \chi u \in H^{1}(\Omega) \text { for all } \chi \in \mathcal{D}\left(\mathbb{R}^{3}\right)\right\} \\
\Delta u(\mathbf{x})+k^{2} u(\mathbf{x})=0 \text { on } \Omega \\
\frac{\partial u}{\partial n}(\mathbf{x})=-\frac{\partial u_{\text {inc }}}{\partial n}(\mathbf{x}) \text { on } \partial \Omega \\
u(\mathbf{x}) \text { is outgoing. }
\end{array}\right.
$$

Standard notation in the theory of partial differential equations are used without comment.

To numerically solve the aboc problem, a classical approach consist in restricting the computational domain to $\{\mathbf{x} \in \Omega \mid\|\mathbf{x}\|<R\}$ where $R$ is chosen large enough so that $\Omega^{\prime} \subset\left\{\mathbf{x} \in \mathbb{R}^{3}:\|\mathbf{x}\|<R\right\}$. The remove unbounded part is then taken into account by an artificial boundary condition posed on the sphere $\left\{\mathbf{x} \in \mathbb{R}^{3} \mid\|\mathbf{x}\|=R\right\}$.

This impedance boundary condition can either be an exact non local Dirichlet to Neumann map, see [6], or an approximate local differential operator which can be derived by a microlocal analysis, see $[4,5]$.

When the rigid body is elongated, this choice of computational domain is not optimal. To avoid unnecessary computational costs, the authors of $[12,2]$ have proposed to use a prolate spheroidal computational domain:

$$
\mathrm{D}=\left\{\mathbf{x} \in \Omega \mid \frac{x^{2}+y^{2}}{b^{2}}+\frac{z^{2}}{a^{2}} \leq 1\right\} \quad \text { with } b<a .
$$

where $a$ and $b$ are chosen large enough to ensure that the rigid body $\Omega^{\prime}$ is included in the spheroidal domain of semi-major axis $a$ and semi-minor axis $b$. A radiation boundary condition is then posed on the artificial boundary

$$
\Gamma=\left\{\mathbf{x} \in \Omega \mid \frac{x^{2}+y^{2}}{b^{2}}+\frac{z^{2}}{a^{2}}=1\right\}
$$

\section{The radiation condition in spheroidal coordinates}

We take advantage of the properties of the scattered field in the exterior domain $\mathrm{D}^{\prime}=\left\{\mathbf{x} \in \mathbb{R}^{3} \mid \frac{x^{2}+y^{2}}{b^{2}}+\frac{z^{2}}{a^{2}} \geq 1\right\}$ to derive the Dirichlet to Neumann map DtN defined on $\Gamma$ by

$$
\frac{\partial u}{\partial n}+\operatorname{DtN} u=0 \quad \text { on } \Gamma
$$

\footnotetext{
*Projet Magique 3D, INRIA Bordeaux Sud-Ouest

${ }^{\dagger}$ LMA, UMR CNRS 5142, Université de Pau et des Pays de l'Adour

${ }^{\ddagger}$ Basque Center for Applied Mathematics (ㄷM), Bizkaia Technology Park, Building 500, 48160 Derio, Basque Country, Spain
} 
In $\mathrm{D}^{\prime}$, the scattered field $u$ is an outgoing solution of the Helmholtz equation

$$
\left\{\begin{array}{l}
u \in H_{\mathrm{loc}}^{1}\left(\mathrm{D}^{\prime}\right) \text { outgoing } \\
\Delta u+k^{2} u=0 \text { in } \mathrm{D}^{\prime} .
\end{array}\right.
$$

According to [9], [12] or [3], we can perform a separation of variables for the Helmholtz equation using the prolate spheroidal coordinates $\xi \in[1,+\infty[, \eta \in[-1,1]$ and $\varphi \in[0,2 \pi[$

$$
\left\{\begin{array}{l}
x(\xi, \eta, \varphi)=f \sqrt{\left(\xi^{2}-1\right)\left(1-\eta^{2}\right)} \cos (\varphi) \\
y(\xi, \eta, \varphi)=f \sqrt{\left(\xi^{2}-1\right)\left(1-\eta^{2}\right)} \sin (\varphi), \\
z(\xi, \eta, \varphi)=f \xi \eta
\end{array}\right.
$$

where $f>0$ is the interfocal distance of the prolate spheroid $\Gamma$ such that $f^{2}=a^{2}-b^{2}$.

Hence, the function $u$ restricted to $\mathrm{D}^{\prime}$ can be determined analytically up to a family of scalars $u_{m, n} \in \mathbb{C}$ :

$$
u(\mathbf{x}(\xi, \eta, \varphi))=\sum_{(m, n) \in \mathbb{S}} u_{m, n} \frac{\Psi_{m, n}(k f, \xi)}{\Psi_{m, n}(k f, a / f)} \frac{Y_{m, n}(k f, \eta, \varphi)}{\left\|Y_{m, n}\right\|_{\Gamma}^{2}} \quad \text { in } \mathrm{D}^{\prime}
$$

$\mathbb{S}$ is the set of integers such that $\mathbb{S}=\{(m, n) \in \mathbb{Z} \times \mathbb{N}:-n \leq m \leq n\}, \Psi_{m, n}$ are the prolate radial spheroidal functions of third type (see Section 3) and $Y_{m, n}$ are the prolate angular spheroidal functions (see Chapter 21 of [1]) which constitute an orthogonal basis for the weighted $L^{2}$-space

$$
\left\|Y_{m, n}\right\|_{\Gamma}^{2}=\int_{\Gamma} \frac{\left|Y_{m, n}(k f, \eta(\mathbf{x}), \varphi(\mathbf{x}))\right|^{2}}{\sqrt{a^{2} / f^{2}-z^{2} / a^{2}}} d s_{\mathbf{x}}
$$

The scalars $u_{m, n} \in \mathbb{C}$ are related to the trace $u$ on the surface $\Gamma$ through

$$
u_{m, n}=\int_{\Gamma} \frac{u(\mathbf{x}) \overline{Y_{m, n}}(k f, \eta(\mathbf{x}), \varphi(\mathbf{x}))}{\sqrt{a^{2} / f^{2}-z^{2} / a^{2}}} d s_{\mathbf{x}}
$$

Then, the normal derivative of the scattered field $u(\mathbf{x})$ satisfies:

$$
\frac{\partial u}{\partial n}(\mathbf{x})=\frac{b}{f^{2}} \frac{\sum_{(m, n) \in \mathbb{S}} u_{m, n} Z_{m, n}\left(k f, \frac{a}{f}\right) Y_{m, n}(k f, \eta(\mathbf{x}), \varphi(\mathbf{x}))}{\sqrt{a^{2} / f^{2}-z^{2} / a^{2}}\left\|Y_{m, n}\right\|_{\Gamma}^{2}} \text { on } \Gamma
$$

with the impedance coefficient $\left.Z_{m, n}:\right] 0,+\infty[\times] 1,+\infty[\longrightarrow \mathbb{C}$ defined by:

$$
Z_{m, n}(c, \xi)=\frac{\frac{\partial \psi_{m, n}}{\partial \xi}(c, \xi)}{\psi_{m, n}(c, \xi)} \quad \text { for all }(m, n) \in \mathbb{S}
$$

Proposition 1. The non local Dirichlet to Neumann operator DtN : $H^{\frac{1}{2}}(\Gamma) \longrightarrow H^{-1 / 2}(\Gamma)$ has the following expression

$$
\operatorname{DtN}: v \longmapsto-\frac{b}{f^{2}} \frac{\sum_{(m, n) \in \mathbb{S}} v_{m, n} Z_{m, n}\left(k f, \frac{a}{f}\right) Y_{m, n}(k f, \eta(\mathbf{x}), \varphi(\mathbf{x}))}{\sqrt{a^{2} / f^{2}-z^{2} / a^{2}}\left\|Y_{m, n}\right\|_{\Gamma}^{2}} .
$$

with $v_{m, n} \in \mathbb{C}$ as $v_{m, n}$ in (9) but replacing $u$ by $v$.

Hence, the restriction to $\mathrm{D}$ of the scattered field solves the following mixed problem:

$$
\left\{\begin{array}{l}
u \in H^{1}(\mathrm{D}), \\
\Delta u+k^{2} u=0 \quad \text { in } \mathrm{D}, \\
\frac{\partial u}{\partial n}=-\frac{\partial u_{\mathrm{inc}}}{\partial n} \text { on } \partial \Omega, \\
\frac{\partial u}{\partial n}+\operatorname{DtN} u=0 \quad \text { on } \Gamma .
\end{array}\right.
$$

In this work we investigate the properties of the coefficients $Z_{m, n}(k f, a / f)$ to develop an accurate numerical method for their computation. Then, we state the well-posedness of problem (13) and propose a variational formulation adapted to the finite element solution of the initial scattering problem. 


\section{The impedance coefficients}

Let $c>0$ and $(m, n) \in \mathbb{S}$. The radial spheroidal functions of third type $\psi_{m, n}(c, \cdot) \in C^{\infty}(] 1,+\infty[)$, see [1], solve

$$
\left\{\begin{array}{l}
\frac{\partial}{\partial \xi}\left(\left(\xi^{2}-1\right) \frac{\partial \psi_{m, n}}{\partial \xi}\right)(c, \xi)+\left(c^{2} \xi^{2}-\frac{m^{2}}{\xi^{2}-1}-\lambda_{m, n}(c)\right) \psi_{m, n}(c, \xi)=0, \\
\frac{d \psi_{m, n}}{d \xi}(c, \xi)-i c \psi_{m, n}(c, \xi)=\underset{\xi \rightarrow+\infty}{O}\left(\frac{1}{\xi^{2}}\right),
\end{array}\right.
$$

where the scalars $\lambda_{m, n}(c) \in \mathbb{R}$ are the so-called prolate spheroidal eigenvalues (see [1]). These systems uniquely define the radial spheroidal functions up to a multiplicative constant which can be chosen arbitrarily. Among all the possible normalizations, we fix the multiplicative constant by imposing the asymptotic expansion of $\psi_{m, n}$ at infinity such that

$$
\psi_{m, n}(c, \xi)=\frac{\exp (i c \xi)}{\xi}(1+\underset{\xi \rightarrow+\infty}{o}(1)) .
$$

Taking into account (11), (14) and (15) the function $Z_{m, n}(c, \xi)$ can be seen as the unique solution of the generalized Riccati equation

$$
\left\{\begin{array}{l}
\partial_{\xi} Z_{m, n}(c, \xi)+F_{m, n}\left(Z_{m, n}(c, \xi), \xi\right)=0 \quad \forall \xi>1 \\
\lim _{\xi \rightarrow+\infty} Z_{m, n}(c, \xi)=i c
\end{array}\right.
$$

with the function $\left.F_{m, n}: \mathbb{C} \times\right] 1,+\infty[\longrightarrow \mathbb{C}$ given by

$$
F_{m, n}(Z, \xi)=Z^{2}+\frac{2 \xi}{\xi^{2}-1} Z+\left(c^{2}+\frac{c^{2}-\lambda_{m, n}(c)}{\xi^{2}-1}-\frac{m^{2}}{\left(\xi^{2}-1\right)^{2}}\right) .
$$

The following lemma is crucial for proving that Problem (13) is well-posed.

Lemma 1. For all $(m, n) \in \mathbb{S}$, we have

$$
\Im Z_{m, n}(c, \xi)>0 .
$$

Proof. Let $R$ be the subset

$$
R=\{\xi \in] 1,+\infty\left[: Z_{m, n}(\xi) \text { is real }\right\} .
$$

We will prove that $R=\emptyset$ by showing that $R$ is closed and open with respect to the canonical topology of ] $-1,+\infty[$. Since $Z_{m, n}$ is continuous, $R$ is closed. Let us consider $\left.\xi_{*} \in\right] 1, \infty\left[\right.$ such that $Z_{m, n}\left(c, \xi_{*}\right)$ is real. Let us remark that $F_{m, n}(Z, \xi)$ is real for all $\left.\xi \in\right] 1,+\infty\left[\right.$ and $Z$ real . Consequently $Z_{m, n}(\xi)$ is real in a neighborhood of $\xi_{*}$ due to the Cauchy Lipschitz Picard theorem. Hence, we have $R=] 1+\infty\left[\right.$ or $\emptyset$. We conclude that $R=\emptyset$ since $Z_{m, n}$ is not real for sufficiently large $\xi$.

Equation (16) is posed on an unbounded domain which should be truncated and a terminal value is thus required to compute a numerical approximation of $Z_{m, n}(c, \xi)$. We define a zero order approximation as the unique solution to the problem

$$
\left\{\begin{array}{l}
\left.Z_{m, n}^{\mathrm{app}}:\right] 0,+\infty\left[\times\left[1, \xi_{\text {max }}\right] \longrightarrow \mathbb{C}\right. \\
\left.\left.\partial_{\xi} Z_{m, n}^{\mathrm{app}}(c, \xi)+F_{m, n}\left(Z_{m, n}^{\mathrm{app}}(c, \xi), \xi\right)=0 \quad \forall \xi \in\right] 1, \xi_{\text {max }}\right] \\
Z_{m, n}^{\mathrm{app}, 0}\left(c, \xi_{\text {max }}\right)=i c .
\end{array}\right.
$$

with $\xi_{\max }>1$ the truncation parameter. Mimicking the method of $[8,7]$, this function can then be numerically computed by an ODE solver backward in time. To be accurate, this approximation needs a large truncation parameter $\xi_{\max }$ which makes it difficult to control the error of the numerical ODE solver. Hence, we are led to improve the choice of the terminal value.

The following lemma is obtained by injecting a generalized Taylor expansion in (16).

Lemma 2. For all $(m, n) \in \mathbb{S}, c>0$ and $p \in \mathbb{N}$, we have the asymptotic expansion:

$$
Z_{m, n}(c, \xi)=Z_{m, n}^{a s y m, p}(c, \xi)+\underset{\xi \rightarrow+\infty}{O}\left(\frac{1}{\xi^{p+1}}\right) .
$$

with

$$
\left\{\begin{aligned}
Z_{m, n}^{\mathrm{asym}, 0}(c, \xi) & =i c \\
Z_{m, n}^{\mathrm{asym}, 1}(c, \xi) & =i c-\frac{1}{\xi} \\
Z_{m, n}^{\mathrm{asym}, 2}(c, \xi) & =i c-\frac{1}{\xi}+\frac{\lambda_{m, n}(c)-c^{2}}{2 i c} \frac{1}{\xi^{2}} \\
Z_{m, n}^{\mathrm{asym}, 3}(c, \xi) & =i c-\frac{1}{\xi}+\frac{\lambda_{m, n}(c)-c^{2}}{2 i c} \frac{1}{\xi^{2}}-\frac{\lambda_{m, n}(c)+c^{2}}{2 c^{2}} \frac{1}{\xi^{3}}
\end{aligned}\right.
$$


The last lemma reveals the terminal value of the $p$-order $(p \in \mathbb{N})$ approximation of $Z_{m, n}$ as

$$
\left\{\begin{array}{l}
\left.Z_{m, n}^{\mathrm{app}, p}:\right] 0,+\infty\left[\times\left[1, \xi_{\text {max }}\right] \longrightarrow \mathbb{C}\right. \\
\left.\left.\partial_{\xi} Z_{m, n}^{\mathrm{app}, p}(c, \xi)+F_{m, n}\left(Z_{m, n}^{\mathrm{app}, p}(c, \xi), \xi\right)=0 \quad \forall \xi \in\right] 1, \xi_{\max }\right] \\
Z_{m, n}^{\mathrm{app}, p}\left(c, \xi_{\max }\right)=Z_{m, n}^{\mathrm{asym}, p}\left(c, \xi_{\max }\right) .
\end{array}\right.
$$

\section{Computation of the spheroidal functions}

In these simulations, the parameter $c=k f$ is fixed to $10 \sqrt{0.75}$. We compare the zero order, first order, second order and third order approximations of $Z_{m, n}$. These approximations are numerically computed with a backward in time RK4 method with a timestep $h=0.01$. For some modes $(m, n) \in \mathbb{S}$, their real and imaginary parts can be found in Figure 1, 2, 3 and 4 . The truncature parameter is given as $\xi_{\max }=4$.
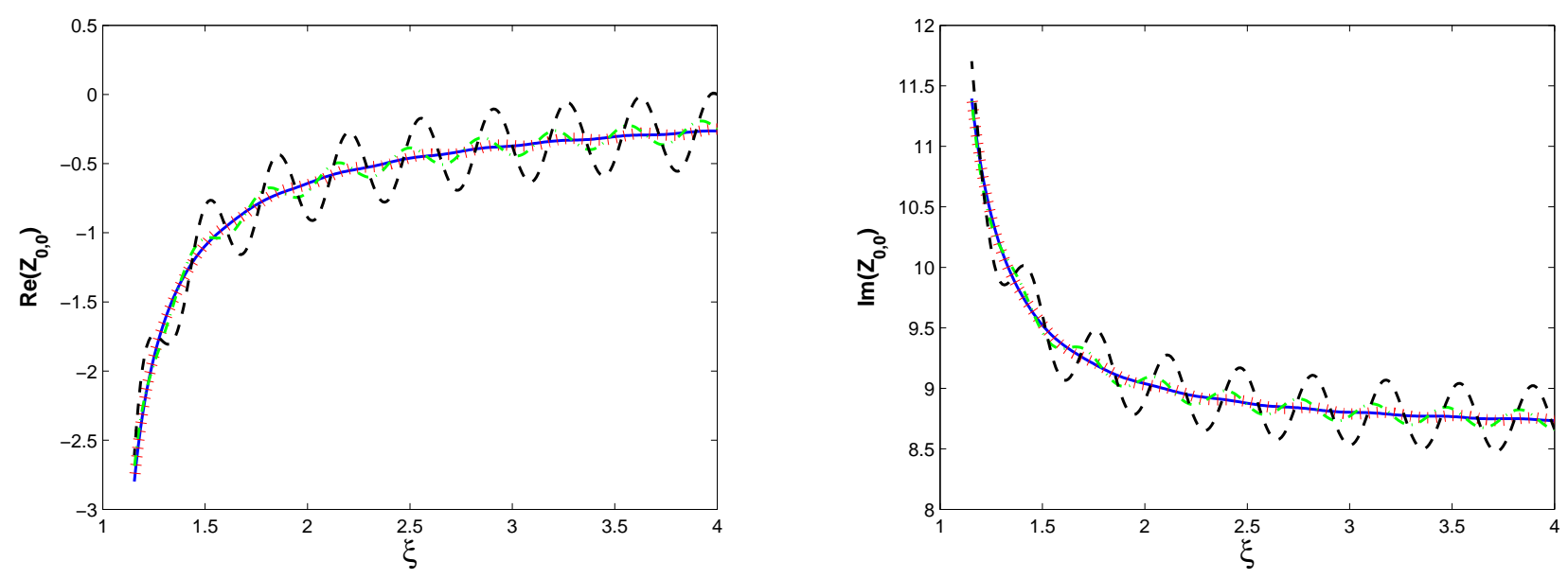

Figure 1: Real and imaginary parts of $Z_{0,0}^{a p p, p}$ for $p=0(--), p=1(---), p=2(|| \mid)$ and $p=3(-)$.
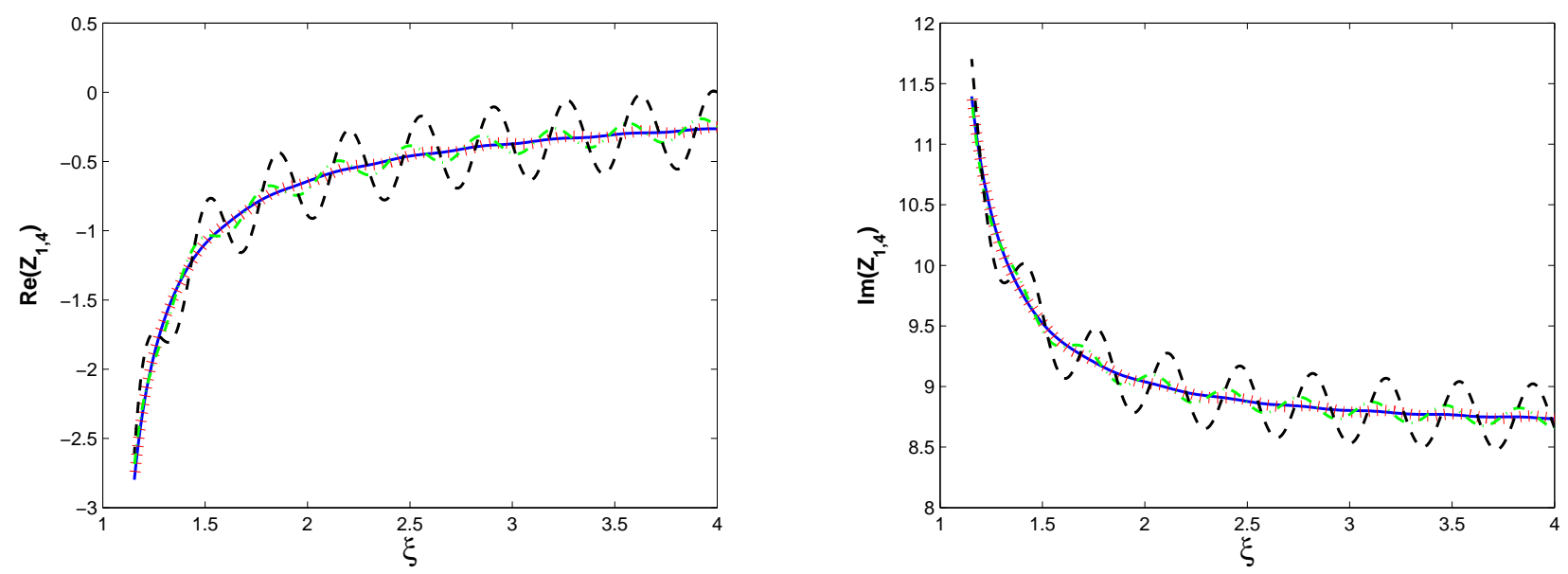

Figure 2: Real and imaginary parts of $Z_{1,4}^{a p p, p}$ for $p=0(--), p=1\left(--_{-}\right), p=2(|| \mid)$ and $p=3(-)$. 

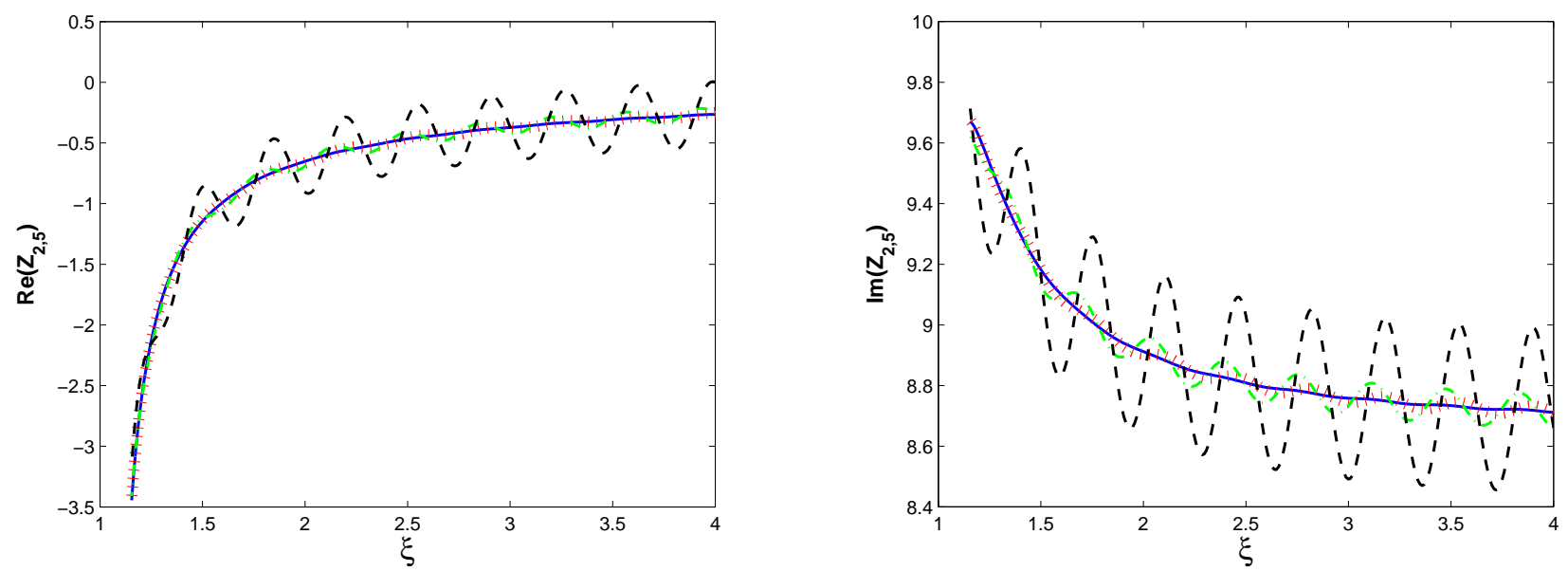

Figure 3: Real and imaginary parts of $Z_{2,5}^{a p p, p}$ for $p=0(--), p=1(--), p=2(|| \mid)$ and $p=3(-)$.
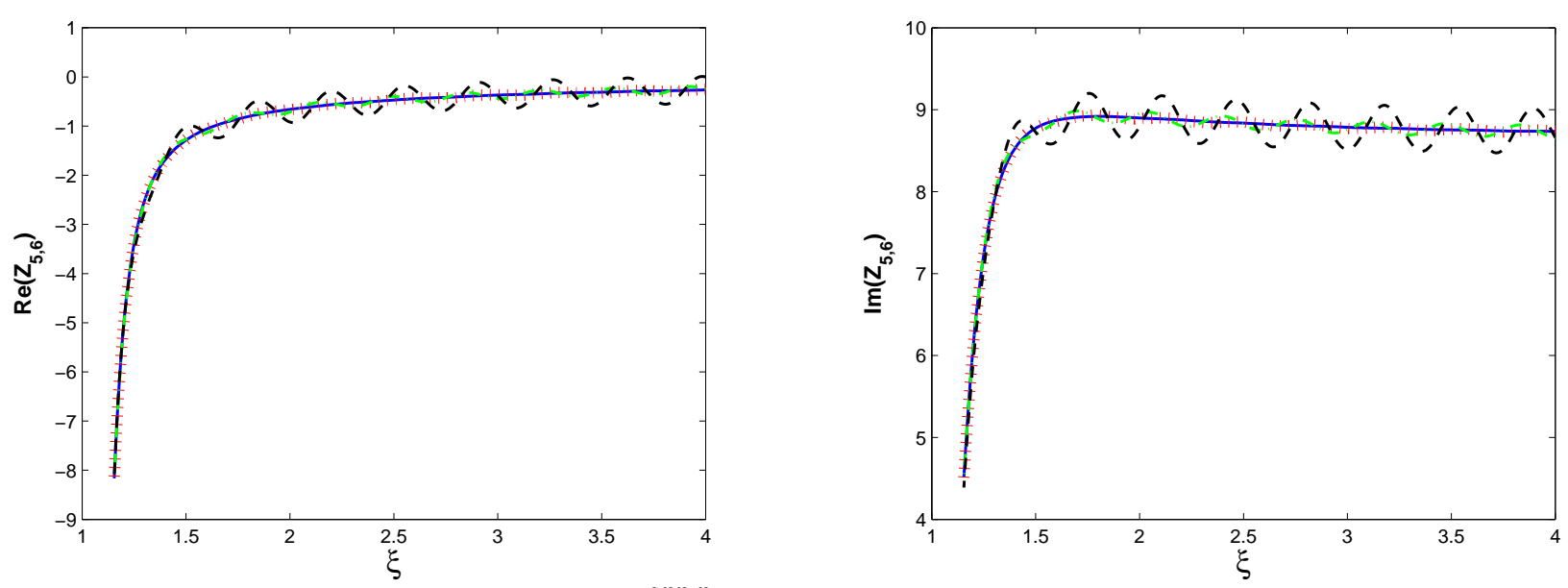

Figure 4: Real and imaginary parts of $Z_{5,6}^{a p p, p}$ for $p=0(--), p=1(--), p=2(|| \mid)$ and $p=3(-)$.

We mainly observe that increasing the order of the approximation reduces the spurious oscillations.

\section{A well-posed variational formulation}

Proposition 2. The restriction to $\mathrm{D}$ of the solution to (1) is the unique function $u \in H^{1}(\mathrm{D})$ satisfying

$$
\underbrace{\int_{\mathrm{D}}\left(\nabla u(\mathbf{x}) \cdot \overline{\nabla v(\mathbf{x})}-k^{2} u(\mathbf{x}) \overline{v(\mathbf{x})}\right) d \mathbf{x}}_{\mathrm{a}(u, v)}+\underbrace{\left(-\frac{b}{f^{2}} \sum_{(m, n) \in \mathbb{S}} Z_{m, n}\left(k f, \frac{a}{f}\right) \frac{u_{m, n} \overline{v_{m, n}}}{\left.\left\|Y_{m, n}\right\|_{\Gamma}^{2}\right)}\right)}_{\mathbf{b}(u, v)}=-\underbrace{-\int_{\partial \Omega} \frac{\partial u_{\mathrm{inc}}}{\partial n}(\mathbf{x}) \overline{v(\mathbf{x})} d s_{\mathbf{x}}}_{\ell(v)}
$$

for all $v \in H^{1}(\mathrm{D})$, with the forms $\mathrm{a}: H^{1}(\mathrm{D}) \times H^{1}(\mathrm{D}) \longrightarrow \mathbb{C}, \mathrm{b}: H^{1}(\mathrm{D}) \times H^{1}(\mathrm{D}) \longrightarrow \mathbb{C}$ and $\ell: H^{1}(\mathrm{D}) \longrightarrow \mathbb{C}$.

Proof. Due to the Fredholm alternative the well-posedness will follow from the uniqueness of the solution. Let us show that $u=0$ in $D$ if $\mathrm{a}(u, v)+\mathrm{b}(u, v)=0$ for all $v \in H^{1}(\mathrm{D})$. Equivalently, the function $u$ solves the following system

$$
\left\{\begin{array}{l}
u \in H^{1}(\mathrm{D}), \\
\Delta u+k^{2} u=0 \quad \text { in } \mathrm{D}, \\
\frac{\partial u}{\partial n}=0 \quad \text { on } \partial \Omega \\
\frac{\partial u}{\partial n}+\operatorname{DtN} u=0 \quad \text { on } \Gamma .
\end{array}\right.
$$


Taking $v=u$ as test function, we have $\Im(\mathrm{a}(u, u)+\mathrm{b}(u, u))=\Im(\mathrm{b}(u, u))=0$. Evaluating this expression, we get

$$
\sum_{(m, n) \in \mathbb{S}_{N}}\left(\Im Z_{m, n}\left(c, \frac{a}{f}\right)\right) \frac{\left|u_{m, n}\right|^{2}}{\left\|Y_{m, n}\right\|_{\Gamma}^{2}}=0 .
$$

Equation (18) leads to $u_{m, n}=0$ for all $(m, n) \in \mathbb{S}$. Then, due to (7), (12) and (25), we have

$$
u=0 \quad \text { and } \quad \frac{\partial u}{\partial n}=0 \quad \text { on } \Gamma .
$$

Finally, the unique continuation principle [11] ensures that $u=0$ in $D$ which concludes the proof.

The bilinear form $b$ involves an infinite number of terms and should be truncated before being implemented. In the following, we denote by $N \in \mathbb{N}$ the order of truncation. In order to avoid the existence of spurious modes, the approach of [10] is adopted. Since $Y_{m, n}$ is an orthogonal basis for the weighted $L^{2}$ space, for all $\alpha \in \mathbb{C}$, we have the identity

$$
\mathrm{b}(u, v)=-\sum_{(m, n) \in \mathbb{S}}\left(\frac{b}{f^{2}} Z_{m, n}\left(c, \frac{a}{f}\right)+\alpha\right) \frac{u_{m, n} \overline{v_{m, n}}}{\left\|Y_{m, n}\right\|_{\Gamma}^{2}}+\alpha \int_{\Gamma} \frac{u(\mathbf{x}) \bar{v}(\mathbf{x})}{\sqrt{\frac{a^{2}}{f^{2}}-\frac{z^{2}}{a^{2}}}} d s_{\mathbf{x}} .
$$

We denote by $\mathbb{S}_{N}=\{(m, n) \in \mathbb{S}: n \leq N\}$. Truncating this expression leads to an approximation $\mathrm{b}_{N}$ of $\mathbf{b}$

$$
\mathrm{b}_{N}(u, v)=-\sum_{(m, n) \in \mathbb{S}_{N}}\left(\frac{b}{f^{2}} Z_{m, n}\left(c, \frac{a}{f}\right)+\alpha\right) \frac{u_{m, n} \bar{v} \overline{m_{m},}}{\left\|Y_{m, n}\right\|_{\Gamma}^{2}}+\alpha \int_{\Gamma} \frac{u(\mathbf{x}) \bar{v}(\mathbf{x})}{\sqrt{\frac{a^{2}}{f^{2}}-\frac{z^{2}}{a^{2}}}} d s_{\mathbf{x}} .
$$

The well-posedness of the truncated variational formulation by reproducing the proof of Proposition 2:

Theorem 1. Let $\alpha \in i \mathbb{R}$ satisfying $\Im \alpha<0$. There exists a unique $u_{N} \in H^{1}(\mathrm{D})$ such that

$$
\mathrm{a}\left(u_{N}, v\right)+\mathrm{b}_{N}\left(u_{N}, v\right)=\ell(v) \quad \forall v \in H^{1}(\mathrm{D})
$$

\section{Acknowledgments.}

This work was supported by the Aquitaine-Euskadi program.

\section{References}

[1] M. Abramowitz and I. A. Stegun. Handbook of Mathematical Functions with Formulas, Graphs, and Mathematical Tables. Dover, New York, ninth dover printing, tenth GPO printing edition, 1964.

[2] H. Barucq, R. Djellouli, and A.-G. Saint-Guirons. Construction and performance assesment of new local DtN conditions for elongated obstacles. Applied Numerical Mathematics, 59 (7):1467-1498, 2009.

[3] X. Claeys. Analyse asymptotique et numérique de la diffraction d'ondes par des films minces. $\mathrm{PhD}$ thesis, Université de Versailles Saint-Quentin-en-Yvelines, December 2008.

[4] B. Engquist and A. Majda. Absorbing boundary conditions for the numerical simulation of waves. Math. Comp., 31:629-651, 1977.

[5] B. Engquist and A. Majda. Radiation boundary conditions for acoustic and elastic wave calculations. Comm. Pure Appl. Math., 32 (3):314-358, 1979.

[6] J. B. Keller and D. Givoli. Exact non-reflecting boundary conditions. Journal of Computational Physics, 82:172192, 1989.

[7] P. Kirby. Calculation of radial prolate spheroidal wave functions of the second kind. Computer Physic Communications, 181:514-519.

[8] P. Kirby. Calculation of spheroidal wave function. Computer Physics Communications, 175:465-472, 2006.

[9] N. N. Lebedev and R. A. Silverman. Special functions and their applications. Dover, New York, 1972.

[10] M. Lenoir and A. Tounsi. The localized finite element method and its application to the two-dimensional seakeeping problem. SIAM J. Numer. Anal., 25:729-752, 1988.

[11] M. H. Protter. Unique continuation for elliptic equations. Trans AMS, 95:81-91, 1960.

[12] A.-G. Saint-Guirons. Construction et analyse de conditions absorbantes de type Dirichlet-to-Neumann pour des frontières ellipsoidales. PhD thesis, Université de Pau et des Pays de l'Adour, November 2008.

[13] C. H. Wilcox. Scattering theory for diffraction gratings. Springer-Verlag, New York, 1984. 